\title{
Hybrid
}

Revue des arts et médiations humaines

$6 \mid 2019$

L'écoute

\section{L'acte compositionnel comme écoute projective : le cas de Trans, pour saxophone ténor et électronique}

\section{Eric Maestri}

\section{(2) OpenEdition}

1 Journals

\section{Édition électronique}

URL : https://journals.openedition.org/hybrid/587

DOI : $10.4000 /$ hybrid.587

ISSN : 2276-3538

Traduction(s) :

Projective listening: Trans, for saxophone and electronics, a case study - URL : https://

journals.openedition.org/hybrid/595 [en]

Éditeur

Presses universitaires de Vincennes

Référence électronique

Eric Maestri, «L'acte compositionnel comme écoute projective : le cas de Trans, pour saxophone ténor et électronique », Hybrid [En ligne], 6 | 2019, mis en ligne le 04 novembre 2019, consulté le 13 avril 2022. URL : http://journals.openedition.org/hybrid/587 ; DOI : https://doi.org/10.4000/hybrid.587

Ce document a été généré automatiquement le 13 avril 2022.

Revue Hybrid 


\title{
L'acte compositionnel comme écoute projective : le cas de Trans, pour saxophone ténor et électronique
}

\author{
Eric Maestri
}

\section{Introduction}

1 Un environnement de travail de studio qui permet de composer d'une manière interactive est à la base de la composition de Trans, pour saxophone ténor et électronique. Ce dispositif permet d'entendre la relation entre la partie électronique et instrumentale directement - comme si on écoutait la pièce - et de plonger l'écoute dans le temps de la musique. Cette approche permet d'inclure l'interprète et de mener un travail collaboratif en utilisant les sons électroniques comme déclencheurs du processus créatif. Progressivement, ce qui était joué en studio est enregistré, fixé et transcrit. Cette fixation implique une projection vers le futur : l'écoute partagée que nous avons menée en studio était orientée vers la performance de la pièce et la préparation de matériel et de supports qui peuvent être interprétés ${ }^{1}$. Cette projection est essentielle dans la pratique compositionnelle : le compositeur, lorsqu'il fait des choix, est obligé de négocier avec l'écoute que d'autres feront de sa pièce. Dans ma pratique compositionnelle, cette forme de projection a une importance particulière. Pour expliquer cette perspective, je me concentre sur trois aspects de la composition de Trans. Je parle de comment l'interprète et moi-même avons réalisé les sons de cette pièce à partir de l'organisation progressive des configurations sonores (i); comment l'organisation de la tension musicale se fonde sur des morphologies sonores spécifiques, caractérisées par la dilatation temporelle de processus sonores simples (ii) ; comment l'idée d'écrire la pièce d'une manière performative en studio se reflète dans la stratégie d'exécution. J'aborde alors la question de la «simulation » et de l'aide qu'elle fournit non seulement à la composition, mais aussi à l'interprétation (iii). Ces trois 
aspects me permettront de détailler trois déclinaisons concrètes de la notion d'« écoute projective ».

\section{$L^{\prime} \ll$ acte compositionnel » comme trace existentielle : I'« écoute projective »}

2 Avant d'affronter la discussion d'exemples tirés de ma pièce Trans, je vais tenter de définir l'«acte compositionnel ", cette définition étant préalable à celle d'« écoute projective ». Je conçois l'«acte compositionnel » comme étant caractérisé par quatre moments fondamentaux : le choix d'un son (i), son positionnement dans le temps (ii), son « com-positionnement " avec d'autres sons (iii) et sa projection (iv). Ces moments ne sont pas chronologiquement distincts, mais cohabitent. Ainsi, le choix, le positionnement d'un son par sa perception singulière, son «com-positionnement » et sa mise en relation, différée, avec le contexte de sa présentation publique - c'est-à-dire sa projection - caractérisent un seul mouvement non divisible. Cette définition s'appuie en partie sur la réflexion menée par Martin Heidegger dans le cinquième chapitre de la première section d'Être et temps ${ }^{2}$. Dans ce chapitre, Heidegger analyse la structure même du Dasein ${ }^{3}$, l'« être-au... » [In-Sein]. Une telle structure possède quatre éléments constitutifs: l'affection [Befindlichkeit] (i), la compréhension [Verstehen] (ii), l'interprétation [Auslegung] (iii) et le discours [Rede] (iv). Cette structure du Dasein met en relief le fait que, en tant qu'êtres humains, nous sommes toujours affectés par un état d'âme [Befindlichkeit] (i) ; que cette affection est comprise en tant que possibilité [Verstehen] (ii); que cette possibilité est conçue selon ses développements possibles [Auslegung] (iii); que ces possibilités sont articulées à l'intérieur d'un horizon de sens dans un contexte donné [Rede]. Or, cette structure qu'Heidegger indique au niveau de «l'être-au... » me semble utile pour définir l'« acte compositionnel » aussi.

3 Le fait de choisir un son est la conséquence d'une affection provoquée par le son luimême (i); le reconnaître et le positionner dans le temps de la composition signifie le comprendre dans son potentiel (ii); cette compréhension implique le «compositionnement» des autres sons aussi, c'est-à-dire l'interprétation du sens du placement d'un son à l'intérieur d'un projet qui s'explicite au fur et à mesure (iii). Le sens de ces choix se fonde sur la projection vers le futur de leur présentation publique. L'écoute de l'autre fait alors partie intégrante de l'«acte compositionnel », car elle concerne le sens qu'on associe aux sons que nous choisissons en tant que compositeurs (iv). Le fait de penser un son dans un certain moment d'une pièce - ou plus généralement le sens d'un choix compositionnel - dérive d'une certaine attribution de sens qui se manifeste à l'écoute, et par l'écoute. Les éléments qui définissent, selon cette perspective, l'«acte compositionnel», constituent une sorte de boucle dans laquelle les sons choisis, leurs positionnements par rapport aux autres, les espaces et les auditeurs possibles constituent un tout cohérent. Composer signifie, d'un côté, mobiliser la sensibilité d'un public possible à partir d'une intention personnelle préparée à l'avance - il s'agit d'une écoute double, concentrée sur le présent de la composition et projetée vers un futur possible - et de l'autre, imaginer un événement sonore différé dans le temps. Il s'agit de "pré-voir» une forme à venir, de "préinterpréter " une musique dans et à partir d'une position singulière individuelle. L'écriture est alors une sorte de trace existentielle inscrite à l'instant [t] et projetée vers l'instant de l'écoute $[\mathrm{t}]^{4}$. 


\section{Composer en tant que performance (différée)}

4 L'environnement de travail que j'ai mis en place pour la composition de Trans permet d'écrire la partie instrumentale et la partie électronique en écoutant leur mixage. Cet environnement nous donne la possibilité de générer des sons de synthèse, de les monter sur une station de travail, d'enregistrer ce que l'interprète joue en fonction de l'électronique, d'écrire la partition, de répéter et de jouer la pièce. J'utilise $C_{s o u n d}{ }^{5}$ pour la synthèse sonore et l'échantillonnage, Logic Pro ${ }^{6}$ et Reaper ${ }^{7}$ comme stations de travail, Finale 8 pour l'écriture de la partition, connecté à la station de travail (ce qui permet de coordonner la lecture de l'électronique et de la partition) et Max ${ }^{9}$ pour l'interprétation et l'interface de performance.

Figure 1

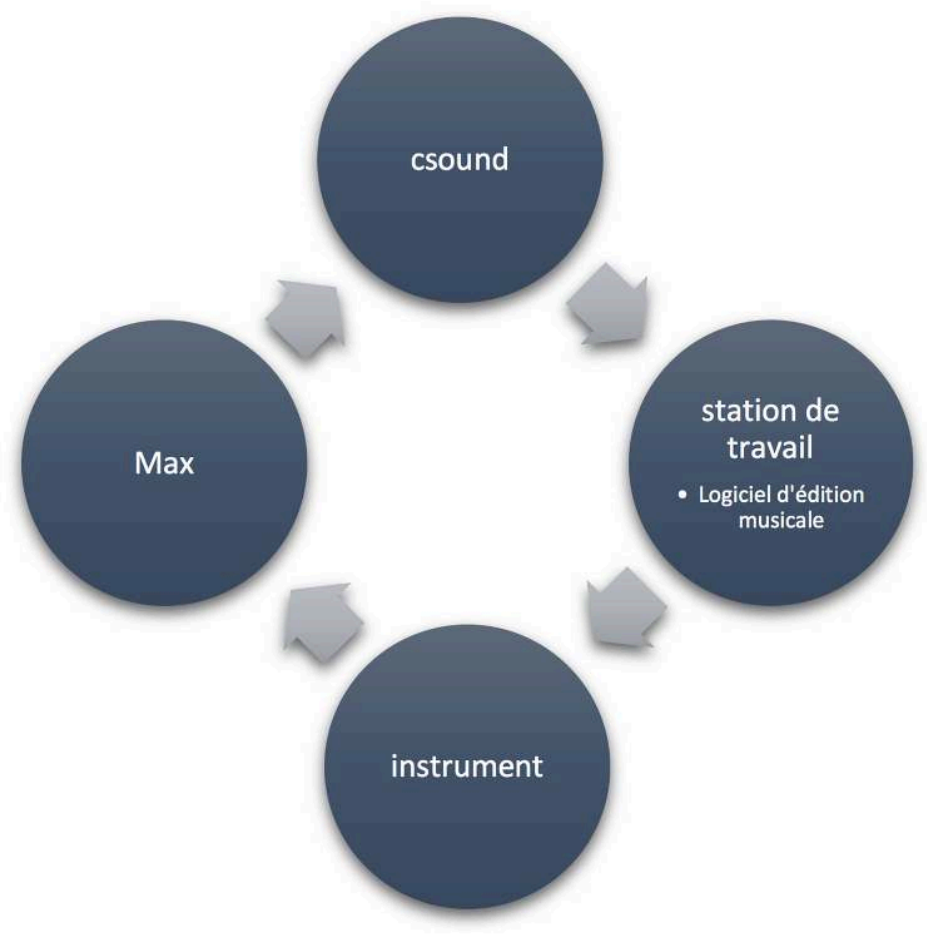

Schéma de l'environnement de travail utilisé pour la composition et l'interprétation de Trans, pour saxophone et électronique.

5 Cet environnement a permis à l'interprète et moi-même de composer d'une manière performative en studio. Cette performance de studio est structurée en quatre moments récursifs :

1. la génération d'un son électronique de synthèse ;

2. le montage des sons de synthèse sur la station de travail et leur mixage. Csound est crucial en ce sens : son rendu très rapide permet une réalisation instantanée des sons électroniques, ce qui permet de suivre le fil des idées musicales par associations en amoindrissant le travail de structuration en amont ;

3. l'improvisation du saxophoniste et l'enregistrement de plusieurs variantes ;

4. la transcription de l'improvisation et la fixation de l'électronique. 
6 La composition des sons électroniques utilise trois instruments de synthèse et un échantillonneur :

Instruments de synthèse utilisés pour la composition de la partie électronique de Trans

\begin{tabular}{|c|c|c|}
\hline $\begin{array}{ll}\text { Type } & \text { de } \\
\text { synthèse }\end{array}$ & $\begin{array}{lr}\text { Instruments } & \text { de } \\
\text { synthèse } & \text { Csound } \\
\text { (opcode) } & \end{array}$ & Description du son \\
\hline Soustractive & Reson & $\begin{array}{l}\text { J'utilise cet instrument pour créer une sonorité brillante } \\
\text { organisée autour de certaines hauteurs définies. }\end{array}$ \\
\hline Additive & Adsynt2 & $\begin{array}{l}\text { Son complexe ayant un nombre élevé de partiels. Cette } \\
\text { synthèse permet de contrôler le rapport entre les } \\
\text { partiels ainsi que leur taux de vibration. }\end{array}$ \\
\hline $\begin{array}{l}\text { Terrain } \\
\text { d'onde }^{10}\end{array}$ & Wterrain & $\begin{array}{l}\text { Cette technique permet de créer des sonorités } \\
\text { complexes à partir du croisement de deux formes } \\
\text { d'ondes, ce qui donne un «terrain d'ondes » qui est } \\
\text { traversé par des trajectoires prédéterminées. }\end{array}$ \\
\hline Échantillonneur & Loscilx & $\begin{array}{l}\text { Il s'agit d'un échantillonneur qui permet de tirer et } \\
\text { transposer des fichiers audios. }\end{array}$ \\
\hline
\end{tabular}

\section{Trois types $\mathrm{d}^{\prime}$ « actes compositionnels » dans Trans}

7 Cinq parties caractérisent Trans. Chacune est définie par une trajectoire, une sonorité, un instrument de synthèse et une technique instrumentale prédominants. Par la notion de «trajectoire », j'entends le passage d'un état de la matière sonore à l'autre en termes de registre (grave et aigu, par exemple) ou de timbre (harmonique ou inharmonique, raréfié ou dense).

8 L'introduction de la pièce a une trajectoire ascendante au niveau du registre et une densification au niveau du timbre. La partie électronique est générée par un procédé de synthèse soustractive. Le saxophone joue un son aérien ${ }^{11}$ autour de la note la 3.

extrait 1

10 La première partie (1) est stable en termes de registre et de timbre : le mouvement est concentré à l'intérieur de la texture et il n'y a pas d'évolution directionnelle de la matière sonore. Dans cette section, comme dans l'introduction, la synthèse numérique prédominante est la soustractive ; le saxophone insiste sur les sons aériens et joue des sons harmoniques à partir de la même fondamentale la 3.

extrait 2

12 La deuxième partie (2) est définie par une accumulation sonore graduelle à partir du matériau précèdent. Dans cette section les trajectoires sont multiples : les registres évoluent vers le grave et l'aigu et le timbre passe de la raréfaction à la densité ; le saxophoniste fait évoluer son jeu de notes tenues (aériennes ou timbrées) jusqu'à des traits plus denses en suivant l'accumulation de la partie électronique. Les synthèses 
employées sont la soustractive et l'additive. L'échantillonneur joue des sons de saxophone spécialement enregistrés et transposés. Une voix enregistrée est aussi utilisée.

\section{extrait 3}

La troisième partie (3) est caractérisée par une lente transition vers le registre grave. Dans cette section, la synthèse soustractive est prédominante; des sons environnementaux sont utilisés aussi. La voix qui apparaît pour la première fois dans la section précédente réapparaît ici. Le saxophone accompagne cette descente progressive en jouant des sons multiphoniques ${ }^{12}$ à partir des notes fondamentales de l'électronique.

\section{extrait 4}

La quatrième partie (4) a une trajectoire multiple aussi, en termes de registre et de timbre; la technique de synthèse par terrains d'ondes prédomine. Le saxophoniste accompagne l'électronique en arrière-plan en jouant des trilles microtonaux ${ }^{13}$. La partie instrumentale est traitée électroniquement avec un retard [delay] en temps réel.

\section{extrait 5}

La dernière partie (5) est caractérisée par une trajectoire vers le grave au niveau du registre, articulée par des pulsations issues d'une synthèse soustractive qui ralentissent progressivement. Le saxophone suit cette descente par un long glissando.

extrait 6

\section{Premier acte : mixer}

L'écoute joue un rôle central dans le mixage et l'organisation du son de l'instrument et de l'électronique dans Trans. La composition de cette pièce se fonde sur le mixage a posteriori des sons instrumentaux et électroniques à partir de l'écoute que l'interprète et moi-même avons faite en studio. La première partie de la pièce nous servira d'exemple.

21 Au début de cette section, je mixe trois types de sons : un son électronique généré par un procédé de synthèse soustractive $(i)$, un son de saxophone ténor qui joue des sons d'air avec des harmoniques naturels de la fondamentale jouée (ii) et un son de synthèse additive en arrière-plan (iii).

Le son (i) filtre un bruit blanc autour de trois hauteurs fixes, la 3, la 7 et la 8 :

extrait 7

En écoutant ce son, le saxophoniste rentre en dialogue avec ce dernier. Il fait résonner l'instrument en mettant en relief le son fondamental de l'électronique d'abord - un la 3 - et les harmoniques dans le registre aigu ensuite :

\section{extrait 8}

L'articulation des phonèmes $[\mathrm{hu}]^{14}$ - pour les résonances dans le registre grave - et [hi $]^{15}$ - pour les résonances dans le registre aigu - permet au saxophoniste d'alterner la note fondamentale, la 3, et ses harmoniques, en particulier mi 6. En changeant la forme de sa bouche, le saxophoniste arrive à rendre instables les harmoniques et à les faire osciller. Notamment, l'harmonique mi 6 oscille d'un demi-ton environ vers $f a 6$. 
Figure 2

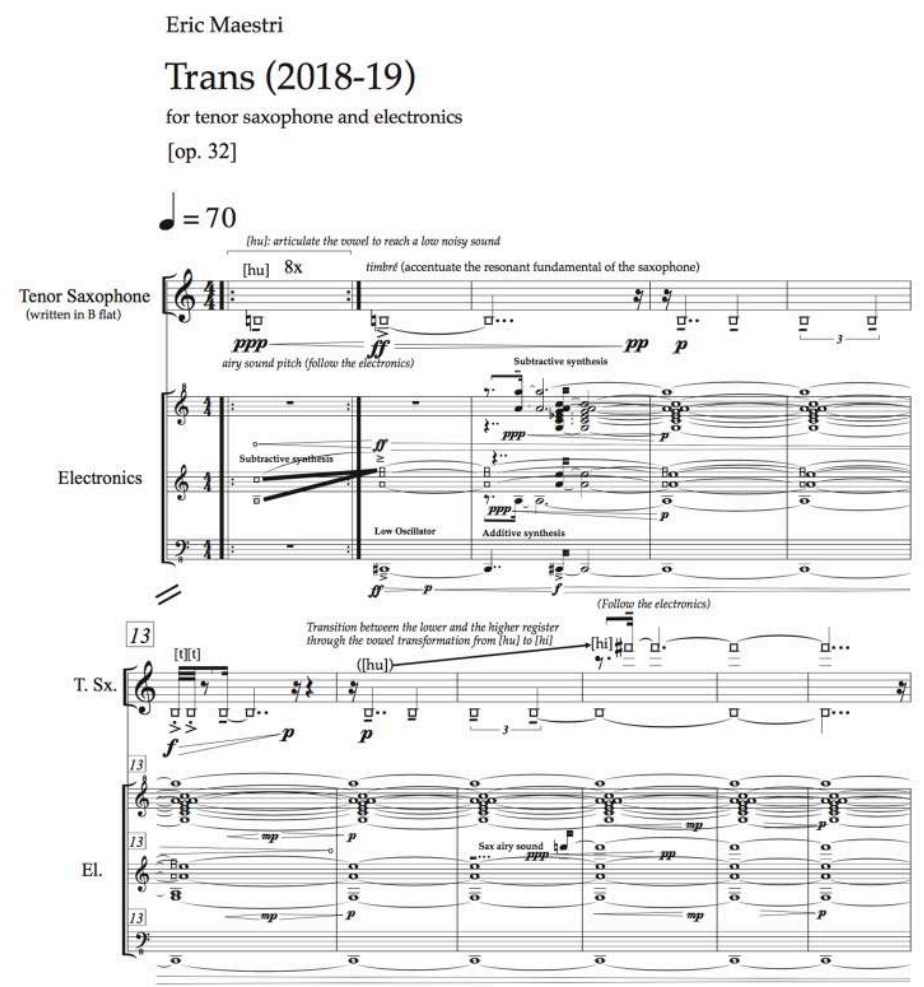

Première page de la partition montrant les techniques de jeu utilisées par le saxophoniste (c) Sugarmusic S.p.A. - Edizioni Suvini Zerboni (avec l'aimable concession de)

Le mixage de ce son instrumental et du son électronique précédent crée une sonorité plus riche :

\section{Extrait 9}

Le travail collaboratif à la base de cette composition permet d'intégrer l'électronique à la partie instrumentale ultérieurement. Notamment, le mixage du son électronique initial et du son instrumental précédent est enrichi par un son de synthèse additive positionné en arrière-plan :

extrait 10

Le mixage des sons électroniques et de la partie instrumentale donne lieu à un son encore plus complexe :

extrait 11

L'organisation du son que nous venons de décrire dérive d'une pratique d'écoute attentive. Le saxophoniste et moi-même avons écouté le premier son de synthèse et avons trouvé une solution instrumentale cohérente. L'écoute du mixage nous y a ultérieurement incités. Ainsi, nous l'avons complété en rajoutant des timbres comme pour composer ensemble. Le rendu sonore final résulte d'un travail d'écriture progressive qui se développe au fur et à mesure grâce à la possibilité d'interagir avec le son directement. À travers le dialogue avec le saxophoniste, j'ai pu fixer des idées musicales claires et les transcrire. Ce procédé, caractérisé par plusieurs allers-retours, permet de jouer avec la pièce et de trouver des configurations sonores spécifiques par tentatives et erreurs. 
34 Le travail collaboratif que j'ai tenté de montrer par cet exemple révèle une fonction première de l'«écoute projective »: l'intégration de l'interprète au niveau de la composition se fonde sur le fait qu'on peut écouter l'œuvre de l'extérieur ensemble. Dans cette situation particulière, nous sommes à la fois acteurs et spectateurs et nous faisons un aller-retour continu entre ces deux dimensions. La dimension performative de cet « acte compositionnel » se fonde sur cette forme d'écoute dédoublée.

\section{Deuxième acte : écouter la tension des trajectoires sonores}

Dans Trans, j'ai cherché à développer des trajectoires musicales linéaires à partir de processus de dilatations temporelles simples, par exemple un glissando d'octave ou un passage graduel de la hauteur au bruit de la durée de trois minutes. Ces exemples définissent des processus musicaux simples dont la dilatation temporelle excessive les transforme en mouvements qui engendrent, selon ma perspective, une tension musicale particulière. Chacune des sections de Trans est définie par un processus de ce genre ayant, comme je l'ai introduit précédemment, une trajectoire unique. Cette trajectoire définit une tension musicale de fond. La première section est caractérisée par une trajectoire stable: l'état sonore de départ correspond, à peu près, à l'état sonore d'arrivée. Cette configuration sonore crée une situation d'attente et d'écoute qui permet à l'auditeur de se concentrer sur les détails internes du son. Les autres parties de la pièce sont fondées sur de lents changements d'état. Par exemple, la deuxième section est caractérisée par l'épaississement graduel de la texture sonore ; la troisième par un glissando progressif vers le registre grave et un épaississement de la matière sonore. Ces trajectoires créent une sorte de bradyséisme sonore qui a l'objectif d'éveiller l'attention et la tension de l'auditeur d'une manière inconsciente. Pour ce faire, j'ai dû me mettre à la place de l'auditeur. J'ai tenté d'imaginer que je portais l'auditeur à la limite de la tension musicale. J'écoutais le processus sonore et j'imaginais quel effet il pouvait avoir sur lui. La durée de ces sections est réalisée d'une manière empirique à travers une multitude d'écoutes orientées pour saisir la durée juste pour obtenir un tel effet. À partir de cette idée, je me suis concentré sur quatre types de mouvements.

Le premier type de mouvement est caractérisé par la transition lente d'un état sonore à l'autre. Notamment, le passage d'une hauteur déterminée au bruit, un crescendo progressif ou un glissando continu d'un registre aigu au plus grave dans une durée longue (par exemple un glissando et crescendo de trois minutes). À partir d'une certaine durée, un mouvement sonore de ce genre devient un fond sur lequel il est possible de composer des articulations plus courtes. C'était le cas pour Trans. Ces mouvements simples de longue durée ont fourni le squelette de la pièce, ensuite articulé par le saxophone et les autres sons électroniques. Ces types de mouvements "subliminaux» accompagnent, comme un véritable bradyséisme, les figures instrumentales et les articulations électroniques plus courtes.

Le deuxième type de mouvement exploite des trajectoires similaires au premier type, mais d'une plus courte durée, par exemple un glissando de trente secondes. Ces mouvements, que j'appelle " liminaux ", restent perceptibles dans leur globalité bien qu'ils soient à la limite des possibilités de la perception et du jeu instrumental. Cette limite m'a particulièrement intéressé. Elle permet de pousser le jeu instrumental jusqu'à l'extrême de ses possibilités lorsqu'on demande à l'interprète de refaire, avec 
son instrument, ce qui est joué par l'électronique. La tension qui s'est créée permet ainsi de définir des configurations sonores paroxystiques. Ce type de mouvement marque le climax de la pièce, dans lequel le son électronique et celui du saxophone suivent la même trajectoire.

Figure 3

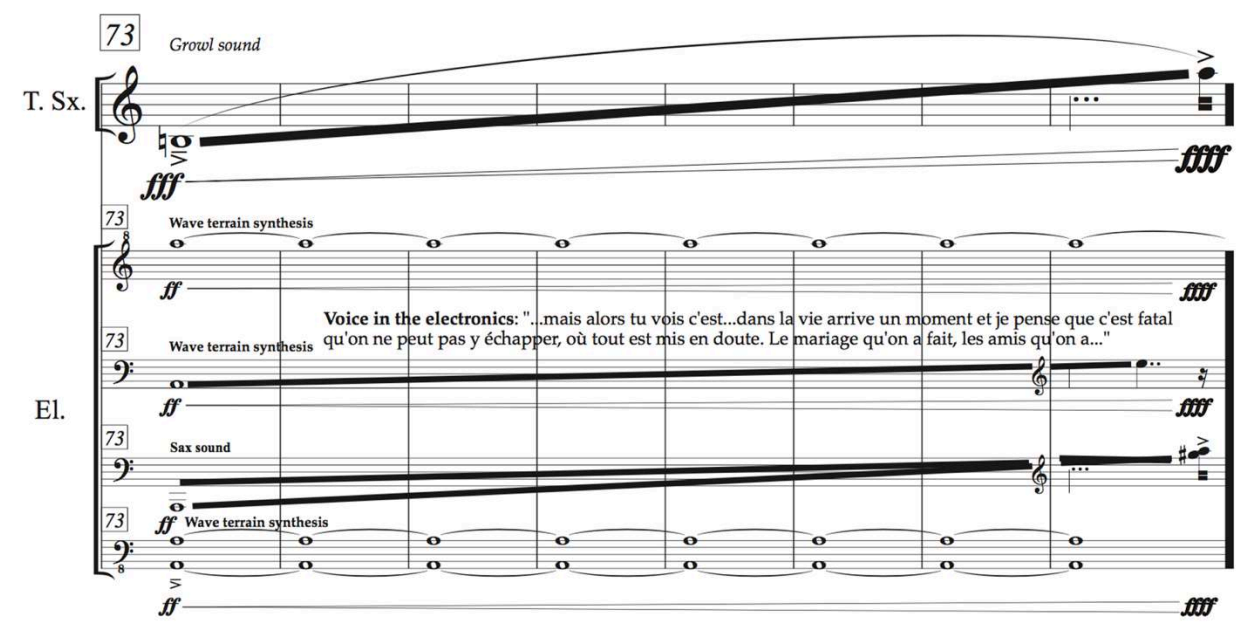

Trait de la pièce qui exemplifie ce que j'appelle mouvement liminal.

(c) Sugarmusic S.p.A. - Edizioni Suvini Zerboni (avec l'aimable concession de)

extrait 13

Le troisième type de mouvement est caractérisé par les articulations et les figures du saxophone. Dans la deuxième section de la pièce, le processus d'accumulation de l'électronique accompagne la densification progressive des traits rapides joués à l'instrument. Cette évolution des modes de jeu se fonde sur le passage graduel des notes aériennes aux notes timbrées jusqu'aux traits rapides et les glissandos violents joués avec une technique dite $"$ growl $»^{16}$.

extrait 14

Le quatrième type de mouvement est caractérisé par des sons environnementaux et des extraits d'entretiens. L'écoute est ainsi portée à faire référence à un champ d'expérience non musical. Les paroles, le son d'une forêt (extrait 15) et d'une rivière (extrait 16) sont mixés dans la pièce.

extrait 15

extrait 16

La conception des trajectoires des processus fondamentaux de Trans se fonde sur une " écoute projective ». Afin de définir leur durée et les types des mouvements, je me suis mis à la place de l'auditeur pour tenter de comprendre le moment dans lequel des processus linéaires de telle sorte dépassent ses possibilités d'attention. J'ai alors pu observer que les processus linéaires lents que j'ai utilisés permettent de produire une tension musicale sous-jacente qui captive l'attention de l'auditeur d'une manière inconsciente. Ce travail d'observation de l'extérieur de la matière sonore profite des possibilités d'écoute multiples; lorsqu'on écoute le même son plusieurs fois dans des conditions d'écoute différentes on commence à le percevoir de l'extérieur, mais en même temps à en apprécier les détails. Ce dédoublement de l'écoute est au cœur de 
l'approche compositionnelle que je décris dans cet article ; il est proche de la notion de "deep listening» que la compositrice américaine Pauline Oliveros avait développé ${ }^{17}$. Cette notion mettait davantage en relief l'importance de l'écoute comme pratique qui éveille la conscience grâce à l'attention portée aux phénomènes sonores. D'une manière similaire, le genre d'écoute attentive que je pratique, qui observe le son en soi et l'horizon de ses écoutes possibles en même temps, tente d'élargir la conscience de mon écoute grâce à la projection que je fais vers les limites de l'autre qui l'écoutera.

\section{Troisième acte : performer une « image-cible »}

L'approche performative qui caractérise l'écriture de Trans a l'objectif de créer un support pour l'exécution. Une fois la partition écrite, nous avons réalisé une maquette de la pièce en mixant l'enregistrement de la partie instrumentale et de la partie électronique. Cette maquette nous a donné une « image sonore » de la pièce qui nous a servi d'« image-cible », c'est-à-dire comme support de la mémoire. extrait 17

L'écoute de cette «image-cible» nous a aidés à retrouver l'équilibre sonore de la simulation durant le concert. L'« image-cible » est une sorte de "partition sonore » qu'on mémorise, qui guide la performance; elle est une sorte de trace qui reste dans la mémoire des interprètes et qui est rappelée durant la performance ou en préparation de la présentation publique. Grâce à l'écoute de cette "image-cible» nous pouvons répéter la pièce en nous mettant à la place de l'auditeur et imaginer son résultat durant la présentation publique. Nous nous projetons alors directement vers l'écoute de l'autre en essayant de le vivre d'abord par une écoute en studio puis pendant les répétitions.

L'« image-cible » est différente du matériel préparé pour le concert. Ce dernier est conçu pour être interprété et donc modifié durant la performance. Pour ce faire, la partie électronique était mixée d'une manière spécifique : j'ai tenté de maintenir une certaine largeur de bande du spectre sonore afin de pouvoir l'égaliser dans la salle et l'adapter en fonction du dispositif disponible. Le travail autour des niveaux sonores et des mélanges entre la partie électronique et instrumentale commencé en studio continue ainsi en concert. Pour réaliser la performance, j'ai préparé un patch Max qui permet de régler le volume et l'égalisation de la partie électronique ainsi que le volume de la partie instrumentale en direct. À ces contrôles, j'ai ajouté un effet de retard [delay] sur le saxophone géré en direct aussi. Ces paramètres me permettent d'interpréter la pièce avec le saxophoniste durant le concert. Un métronome visuel donne au saxophoniste la possibilité de se coordonner avec la partie fixée sur support. L'interprète le regarde par son smartphone posé sur le pupitre. Connecté à un réseau local, il visualise les signaux du métronome provenant du patch $M a x^{18}$. 


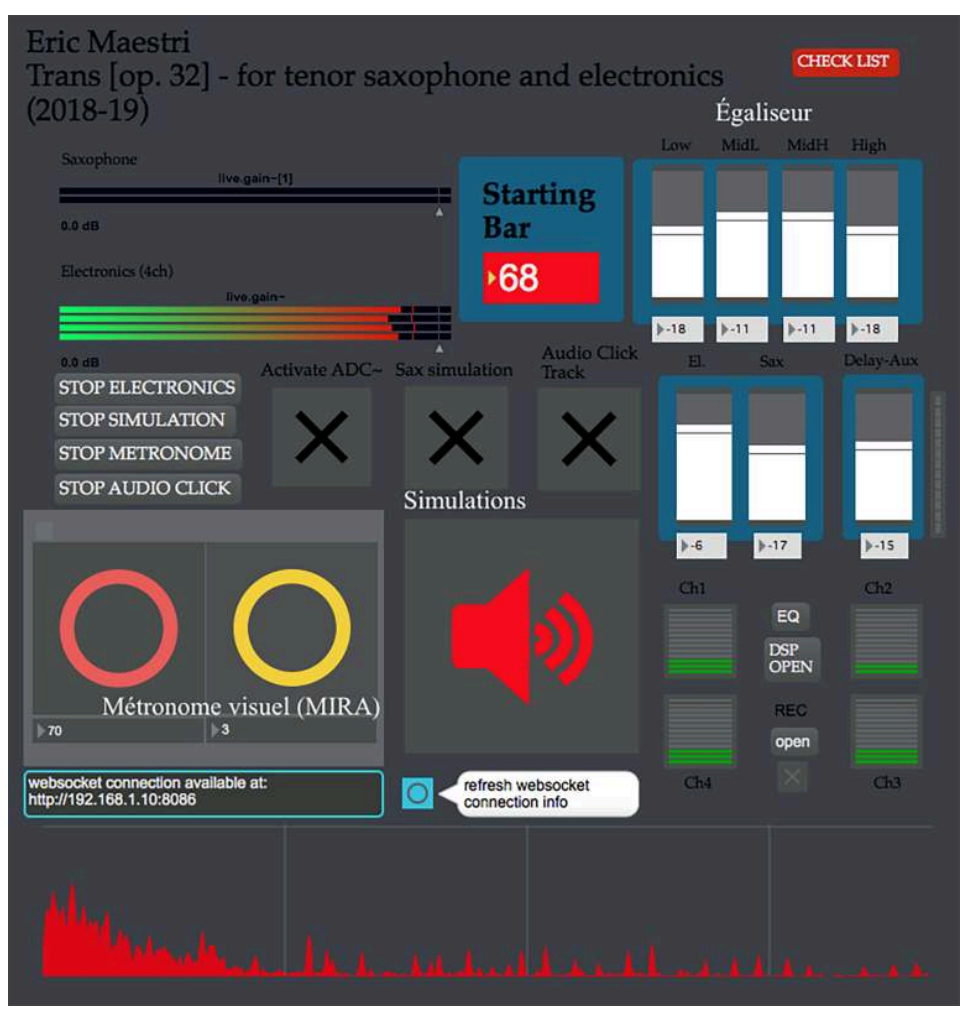

Premier niveau du patch Max. En haut à droite, l'égaliseur et les volumes de l'électronique et de l'instrument. En bas à droite, on observe les niveaux de sortie de l'électronique. Le sonagramme en bas permet de visualiser le résultat de l'action de l'égaliseur. Au centre à gauche, on voit le métronome et l'adresse de connexion qui sert au saxophoniste pour se repérer durant la performance.

Cette approche de l'interprétation montre un troisième aspect de l'«écoute projective ». La simulation de la pièce est conçue pour fournir une «image-cible». Cette « image-cible» nous permet d'écouter la pièce comme si nous étions parmi les auditeurs. Il s'agit d'un exercice de projection qui caractérise tout le processus créatif qui, dans ce dernier cas, permet de préparer la performance et de nous projeter vers l'écoute de l'autre de la manière la plus proche de la réalité du concert. Lors des répétions et du concert, nous nous sommes servis de cette image pour pouvoir nous orienter dans l'interprétation et garder bien clair l'objectif de la performance. Ainsi, le mixage est orienté pour la préparation de l'exécution; il n'a pas comme objectif de réaliser un objet fini, mais plutôt de préparer un matériel ouvert à l'interprétation.

\section{Conclusion}

Dans cet article, j'ai défini l'« acte compositionnel » comme la condition existentielle du compositeur : le compositeur doit choisir des sons, des instruments ou des espaces en fonction d'un projet qui se réalisera dans le futur. Il prépare, pour ainsi dire, l'écoute des autres. La notion d'" acte compositionnel» souligne le fait que les choix compositionnels résultent d'une négociation entre l'identification d'une idée musicale significative pour le compositeur et le sens que cette idée a une fois qu'elle est organisée dans une composition. Cette condition fondamentale développe une «écoute projective » : l'écoute qu'on pratique durant la composition prépare des matériels qui peuvent être manipulés, interprétés et écoutés dans des situations que le compositeur 
ne peut pas prévoir entièrement. L'« acte compositionnel » est alors caractérisé par ce genre d'écoute dédoublée, qui prend en compte l'horizon du futur possible.

J'ai tenté de montrer comment cette " écoute projective » caractérise certains aspects de ma pratique compositionnelle. J'ai choisi de le faire en prenant en compte trois aspects qui concernent ma pièce pour saxophone ténor et électronique Trans. D'abord, la notion d'"écoute projective" indique que lorsqu'on compose en studio d'une manière performative on est à la fois compositeur et auditeur. Ce dédoublement de l'écoute nous permet de partager la composition et de collaborer avec l'interprète durant la composition, comme j'ai pu le montrer dans mon cas; ensuite, l'« écoute projective » concerne ce que j'imagine de l'auditeur, notamment son attention. Cette projection était pour moi fondamentale pour composer Trans, car, dans cette pièce, j'ai cherché à imaginer des processus linéaires de dilatation temporelle simple qui engendrent une tension d'écoute spécifique chez l'auditeur. Pour décider de leur durée, j'ai dû me mettre à la place de l'auditeur, les écouter à plusieurs reprises et progressivement arriver à une solution; enfin, l'« écoute projective » caractérise une approche de la simulation sonore de la pièce. J'ai utilisé la notion d'« image-cible » pour montrer comment la simulation permet d'envisager le résultat de l'exécution en amont. Cette «image-cible » est une sorte de partition sonore, un aide-mémoire et une projection vers la réalité du concert qui conditionne le travail compositionnel et supporte la préparation de la performance.

La discussion de ces aspects de la composition de Trans montre que l'«acte compositionnel » est aussi un acte de projection fondé sur une écoute dédoublée. Je suis alors de l'avis qu'imaginer un dialogue futur avec l'auditeur et l'interprète caractérise profondément la pratique compositionnelle. Cette pratique n'est pas un acte isolé, mais est constituée par un acte dialogique différé dans le temps.

BIBLIOGRAPHIE

Heidegger, Martin, Sein und Zeit, Tuebingen, Max Niemeyer Verlag, 1927.

Maestri, Eric, « Notation as Temporal Instrument », in Nash, Chris, Hoadley, Richard et Fober, Dominique (dir.), Proceedings TENOR 2016. International Conference on Technologies for Music Notation and Representation, Cambridge, Anglia Ruskin University, 2016.

Oliveros, Pauline, Deep Listening: A Composer's Sound Practice, Deep Listening Publications, 2004.

Roads, Curtis, L'Audionumérique. Musique et informatique, traduction Jean de Reydellet, troisième édition, Paris, Dunod, 2016.

Small, Christopher, Musicking. The Meaning of Performing and Listening, Middletown, Wesleyan University Press, 1998.

Weiss, Marcus et Netti, Giorgio, The Techniques of Saxophone Playing, Kassel, Baerenreiter, 2015. 


\section{NOTES}

1. Comme le soutenait Christopher Small, le travail compositionnel prépare le matériel pour la performance. Cf.Christopher Small, Musicking. The Meaning of Performing and Listening, Middletown, Wesleyan University Press, 1998, p. 9 : " To music is to take part, in any capacity, in a musical performance, whether by performing, by listening, by rehearsing or practising, by providing material for performance (what is called composing), or by dancing. "

2. Martin Heidegger, Sein und Zeit, Tuebingen, Max Niemeyer Verlag, 1927, p. 28-38.

3. Le Dasein est le terme choisi par Heidegger pour indiquer l'être humain, c'est-à-dire l'être dont l'existence est sa modalité spécifique d'être.

4. Eric Maestri, "Notation as Temporal Instrument», in Chris Nash, Richard Hoadley et Dominique Fober (dir.), Proceedings TENOR 2016. International Conference on Technologies for Music Notation and Representation, Cambridge, Anglica Ruskin University, 2016.

5. Langage de programmation pour la synthèse sonore; le logiciel libre Csound hérite de la tradition des premiers logiciels de synthèse inventés par Max Mathews (MUSIC-N) et est développé - à partir de 1985 - par Barry Vercoe au Massachussetts Institut of Technology (MIT). Le logiciel utilise des modules appelés «opcode» ou «ugen» (unit generators), c'est-à-dire des générateurs sonores préprogrammés qui peuvent être connectés entre eux et joués en utilisant la syntaxe du logiciel. [https://csound.com/], consulté le 15 juillet 2020.

6. Logiciel commercial de production musicale. L'interface se présente comme un séquenceur multipiste ; cette station de travail permet de monter, traiter et éditer des fichiers de sons et de contrôler des instruments virtuels de synthèse sonore. [https://www.apple.com/logic-pro/], consulté le 15 juillet 2020.

7. Logiciel adapté à la création et à la spatialisation sonores. Cette station de travail offre des possibilités en termes de mixage multicanal. [https://www.reaper.fm/index.php], consulté le 15 juillet 2020.

8. Logiciel de gravure musicale qui peut être mis en connexion avec la station de travail pour coordonner leur lecture.

9. Langage visuel de programmation développé à l'origine par le mathématicien et compositeur Miller Puckette - autour de 1985 - à l'Ircam. Ce logiciel propose, comme Csound, une interface graphique qui permet de visualiser les modules et de les connecter entre eux en créant des programmes, dits « patchs ».

10. Curtis Roads, L'Audionumérique. Musique et informatique, traduction Jean de Reydellet, troisième édition, Paris, Dunod, 2016, p. 415.

11. Le saxophoniste souffle dans le saxophone pour faire résonner la note sélectionnée. Cette technique instrumentale permet de produire un bruit coloré - ayant des fréquences qui apparaissent - qui fusionne avec la partie électronique.

12. Il s'agit de sons instrumentaux complexes caractérisés par plusieurs hauteurs joués sur des instruments monophoniques ; ils s'obtiennent avec l'utilisation de doigtés spécifiques. Dans Trans je fais référence au livre de Marcus Weiss et Giorgio Netti sur les techniques contemporaines pour le saxophone: Marcus Weiss et Giorgio Netti, The Techniques of Saxophone Playing, Kassel, Baerenreiter, 2015.

13. Le saxophone n'est pas construit pour jouer des intervalles microtonales (plus petit du demiton), par conséquent le saxophoniste cherche des doigtés spéciaux qui lui permettent de créer une sorte de vibrato dont la vibration est gérée par le mouvement de certaines clés de l'instrument.

14. 'hu: en alphabet phonétique international

15. 'haI en alphabet phonétique international

16. Technique qui consiste à jouer une note et à chanter une note grave en même temps.

17. Pauline Oliveros, Deep Listening: A Composer's Sound Practice, Deep Listening Publications, 2004.

Hybrid, 6 | 2019 
18. Pour ce faire j'ai utilisé la librairie pour Max miraweb. En ligne: [https://github.com/ Cycling74/miraweb], consulté le 15 juillet 2020.

\section{RÉSUMÉS}

Dans cet article, je discute la notion d'« écoute projective ». À travers cette notion, je me propose de montrer que l'«acte compositionnel» est caractérisé par une forme d'écoute différée, projetée vers les écoutes possibles des auditeurs futurs; il s'agit d'une écoute dédoublée qui intègre dans un seul acte l'instant de la composition et de sa réception. Composer peut signifier, en partie, imaginer l'écoute de l'autre; penser les sons et les figures musicales dans une boucle de sens qui concerne l'idée compositionnelle et sa réception. Pour éclaircir cette notion, je me concentre sur trois aspects de ma pratique. Dans ma pièce Trans, pour saxophone ténor et électronique, j'ai tenté de mixer le son de l'instrument et de l'électronique en créant un environnement de travail qui me permettait d'écouter le mixage durant la composition. Cet environnement performatif m'a permis d'inclure l'interprète dans le processus d'écriture et de créer des textures sonores par des processus simples de dilatation temporelle. La projection vers l'autre, au centre de ce travail, concerne la performance aussi. La discussion de ces trois aspects montrera comment l'écoute de l'autre rentre en jeu durant la composition. Ainsi, je propose de penser l'« acte compositionnel » comme étant ouvert et substantiellement projeté vers le futur.

\section{INDEX}

Mots-clés : acte compositionnel, trace, performance différée, musiques électroniques, interaction, cocréation

\section{AUTEUR}

\section{ERIC MAESTRI}

Eric Maestri est compositeur et chercheur. Il est actuellement professeur de composition électroacoustique au conservatorio Niccolò Paganini de Gênes, et chargé de cours à l'université de la Sorbonne. Il mène une recherche artistique plurielle caractérisée par la mise en perspective de son travail par une approche d'autoanalyse réflexive. 